\title{
eJRIEPS
}

Ejournal de la recherche sur l'intervention en éducation physique et sport

Hors-série $\mathrm{N}^{\circ} 2 \mid 2018$

À propos de certaines bases théoriques et pratiques des sports collectifs

\section{Observer et évaluer en sport collectif : des éléments quantitatifs et qualitatifs}

Jean-Francis Gréhaigne

\section{OpenEdition}

Journals

Édition électronique

URL : http://journals.openedition.org/ejrieps/516

DOI : 10.4000/ejrieps.516

ISSN : 2105-0821

Éditeur

ELLIADD

Référence électronique

Jean-Francis Gréhaigne, «Observer et évaluer en sport collectif : des éléments quantitatifs et qualitatifs », eJRIEPS [En ligne], Hors-série № 2 | 2018, mis en ligne le 01 juillet 2018, consulté le 10 décembre 2020. URL : http://journals.openedition.org/ejrieps/516; DOI : https://doi.org/10.4000/ ejrieps.516

La revue eJRIEPS est mise à disposition selon les termes de la Creative Commons Attribution 4.0 International License. 


\section{Observer et évaluer en sport collectif : des éléments quantitatifs et qualitatifs}

Les difficultés de l'évaluation individuelle et collective en sports collectifs sont celles de tout système complexe, à savoir que de nombreux éléments interviennent en interactions. Il faut également tenir compte de l'influence fondamentale du rapport de forces qui peut évoluer au cours d'une même rencontre ou dans des situations d'opposition différentes. Enfin, quelle est la validité de l'évaluation d'un seul élément (le joueur) dans un système qui a sa cohérence (l'équipe) car l'interdépendance des membres d'une même équipe est patente. Aussi, toute situation d'évaluation repose sur un certain nombre de présupposés qui ont évolué avec le temps en fonction des connaissances ou des conceptions en vogue. Au cours du temps, ce modèle est passé d'une appréciation globale du jeu style « œil du maquignon » aux parcours chronométrés, puis aux grilles comportementales.

Après avoir travaillé avec les nomogrammes (Gréhaigne \& Roche, 1993), aujourd'hui pour évaluer on utilise plutôt des situations aménagées en jeu réduit qui combinent des outils quantitatifs et qualitatifs afin de mieux apprécier ce qui se passe réellement sur le terrain. L'évolution des outils évoqués dans ce bref inventaire souligne bien la difficulté d'évaluer en sport collectif, particulièrement quand on met en avant la notion d'opposition (Gréhaigne, Richard, \& Griffin, 2005). Si cette conception semble novatrice du point de vue des conceptions de l'apprentissage, elle pose de réels problèmes pour l'évaluation pratique des acquis. En effet, quelle est la part de chacun dans le succès ou l'échec de l'équipe ? Comment comparer deux équipes qui ne sont pas affrontées ? Quels indicateurs fiables pour évaluer un rapport de forces ? Ainsi, une bonne évaluation de départ vise à faire un bilan des ressources des élèves au début d'un cycle de formation. Ici, l'évaluation formative a pour but de guider l'élève dans son travail scolaire. Elle cherche à situer ses difficultés pour découvrir des procédures qui lui permettront de progresser dans ses apprentissages (Cardinet, 1984). Aussi, dans le cadre de cette évaluation, il faut se livrer à une observation systématique du jeu donc à une véritable étude éthologique du comportement des joueurs pour éviter d'énoncer ce que les élèves ne savent pas faire. Ces études supposent l'observation du joueur en situation d'affrontement chaque fois qu'elle est possible. Des méthodes de mesures objectives de l'activité des sujets et de traitement des données devraient permettre de quantifier les observations et de discerner ce qui dans une activité relève d'une conduite spécifique ou de comportements anecdotiques ou simplement émergents.

L'utilisation de données chiffrées peut consister à enregistrer des comportements jugés significatifs d'une adaptation à une modélisation du jeu (Brau-Antony, \& Cleuziou, 2005 ; Gréhaigne, 1989 ; Mérand, 1984) soit pour l'équipe, soit pour les joueurs. Comme il a déjà été exposé (Gréhaigne, 
2007) pour l'équipe, le simple fait d'analyser les rapports entre le nombre de buts marqués, le nombre de tentatives de tirs et le nombre d'attaques (ce qui revient à compter le nombre d'entrées en possession du ballon pour l'équipe) dans des rencontres de durée constante donne des indications très précieuses. En effet, l'efficacité est une donnée centrale en sport collectif car pour gagner, il faut marquer un point de plus que l'adversaire. Les comportements des joueurs s'inscrivent et s'insèrent dans des configurations du jeu temporaire, illustrant un rapport d'opposition momentané. Pour obtenir des informations encore plus précises sur les conditions de l'affrontement, on a également utilisé différents indices chiffrés. Nous définirons un indice comme une valeur numérique obtenue en mettant en rapport la fréquence d'apparition de deux comportements. Celuici ne doit pas être considéré comme une mesure absolument exacte car le choix des éléments retenus comporte toujours une part d'arbitraire et de subjectivité. Néanmoins, utilisés avec prudence, les indices constituent souvent le seul moyen d'avoir une connaissance approchée sur des phénomènes complexes qui président au jeu et sur leurs conséquences. Enfin, des études plus qualitatives permettront la collecte d'autres données avec, par exemple, la description d'aspects topologiques du jeu en vue obtenir d'autres informations pertinentes sur l'affrontement comme les interactions et les pratiques concrètes des joueurs.

C'est ce que nous allons envisager maintenant.

\section{Des aspects quantitatifs}

Les observations quantitatives sont faites avec des instruments de mesures. Les résultats peuvent être mesurés ou comptés, et toute autre personne qui tente d'évaluer quantitativement la même situation devrait se retrouver avec les mêmes résultats. On vise à mieux connaître l'organisation des partenaires ou des adversaires constitutifs du jeu à un instant donné ou à des instants successifs.

\section{Les séquences de jeu}

A partir des jeux réduits, une bonne façon d'obtenir des données sur le jeu consiste à analyser les séquences de jeu (Dugrand, 1989 ; Gréhaigne, Billard \& Laroche, 1999; Mérand, 1977, 1984, 1990). Celles-ci peuvent être définies comme les échanges de balle entre les joueurs depuis l'entrée en possession du ballon et jusqu'à sa perte par l'équipe (Figure 1). On relève donc tous les échanges de balle entre les joueurs d'une équipe, mais aussi ceux du joueur qui remet la balle en jeu, qui tire les coups francs etc. En un mot, on enregistre tous les événements du jeu (Figure 1) perte de balle ; touche; but ; tir au but ; coup franc ; corner. 


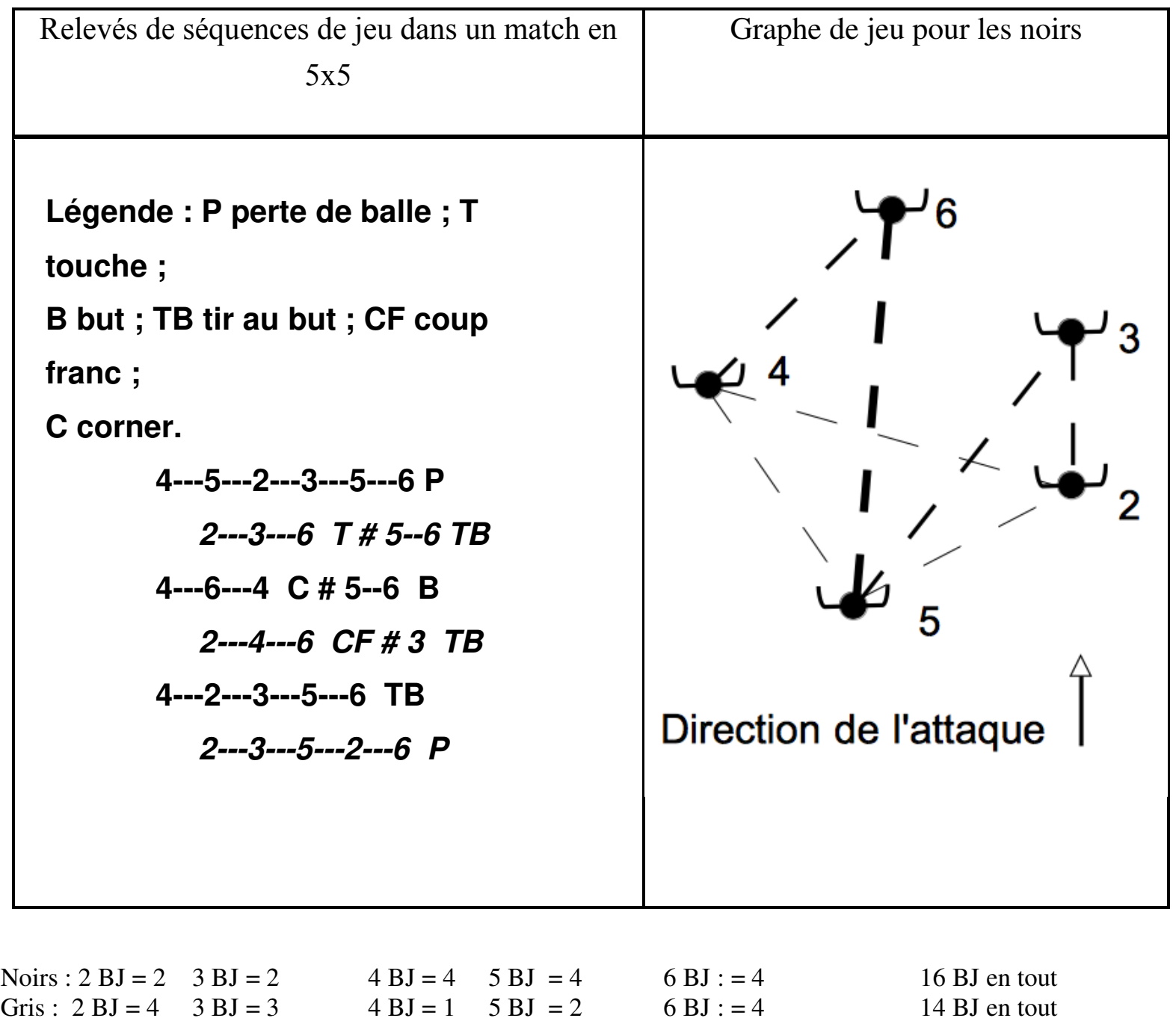

Figure 1. Exemple de relevés chiffrés concernant les échanges de balle

Ces séquences de jeu permettent d'obtenir des renseignements importants comme le nombre de séquences dans un temps donné et le nombre d'échanges de balle à l'intérieur de chaque séquence ainsi que la durée des séquences avant une interruption du jeu. Ce dernier nombre est souvent lié au niveau de l'affrontement. Plus la séquence de jeu est longue, plus l'équipe prouve qu'elle est à même de gérer différents paramètres du jeu, et en particulier le jeu adverse, en enchaînant diverses unités tactiques, ou qu'elle est incapable de pénétrer le dispositif de jeu adverse.

On peut aussi repérer précisément quels sont les joueurs qui font les remises en jeu : engagement, remises de touche, coups francs, quels sont les joueurs qui se contentent de donner le ballon sans risque [notion de balle neutre], qui perdent ou au contraire qui récupèrent des balles), ... et identifier les circulations habituelles de la balle à l'intérieur de l'équipe dans un type d'opposition donné. 
Données chiffrées sur le réseau d'échanges

Une autre façon d'obtenir des données sur le jeu consiste à analyser les séquences de jeu en utilisant les situations à effectif réduit (Dugrand, 1985 ; Luthanen, 1986). Celles-ci peuvent être définies comme les échanges de balle entre les joueurs depuis l'entrée en possession du ballon jusqu'à sa perte par l'équipe (but ou balle reprise par l'adversaire). On relève donc tous les échanges de balle à l'intérieur de l'équipe, mais aussi qui engage, etc. En d'autres termes, on enregistre tous les événements qui se produisent dans le jeu. Dans l'exemple exposé (tableau I et figure 1), les données chiffrées ont été recueillies avec des joueuses du Lycée de Thala en Tunisie, en 5 x 5 pendant cinq minutes en utilisant le handball comme sport collectif support (Zerai, 2011).

En interprétant les données chiffrées fournies par ce type de relevé, on peut repérer précisément qui fait les engagements ou récupère activement le ballon (ici plutôt le numéro 4), qui mène le jeu et fait les remises en touche (le numéro 5), qui se contente de donner des balles neutres (numéro 2), qui perd des balles, qui tire et marque (le numéro 6)... Ces indicateurs sont révélateurs de la distribution préférentielle des rôles au sein de l'équipe.

On peut également construire un schéma des échanges qui permet d'illustrer les circulations de balle privilégiées et les relations entre les joueurs, et ainsi avoir une représentation graphique des circulations habituelles de la balle à l'intérieur de l'équipe et du rapport de compétences dans un type d'opposition donné. Un réseau de compétences est constitué par une distribution des tâches voulues par quelques-uns, des conflits, ou une répartition tacite des rôles et des fonctions.

Une autre utilisation de données chiffrées peut consister à enregistrer des comportements jugés significatifs d'une adaptation à une modélisation du jeu (Gréhaigne, 1992 ; Blomqvist, Vänttinen, \& Luthanen, 2005 ; Brau-Antony, \& Cleuziou, 2005) soit pour l'équipe soit pour les joueurs. Comme nous l'avons déjà exposé (Gréhaigne, 2007) pour l'équipe, le simple relevé du nombre de buts marqués (B), du nombre de tentatives de tirs (T) et du nombre d'attaques (ce qui revient à compter le nombre d'entrées en possession du ballon) dans des rencontres de durée constante, donne des indications très précieuses. On peut également relever les actions positives.

Les actions positives

Les actions positives sont des actions de jeu qui constituent les conduites nouvelles attendues dans les configurations de jeu vécues. 
Tableau I. Des exemples d'actions positives

\begin{tabular}{|c|c|}
\hline Pour les attaquants & Pour les défenseurs \\
\hline $\begin{array}{l}\text { Tirs cadrés, but marqué } \\
\text { Franchir la ligne d'avantage } \\
\text { Réalisation d'un } 1-2 \\
\text { Passe longue réussie à un partenaire en } \\
\text { avant } \\
\text { Passe décisive, centre permettant un tir } \\
\text { Déviation sans contrôle vers l'avant. }\end{array}$ & $\begin{array}{l}\text { Interception volontaire } \\
\text { Duel gagné } \\
\text { Replacement entre le ballon et sa propre } \\
\text { cible } \\
\text { Recul frein pour retarder la progression } \\
\text { Ecarter le ballon sans le récupérer } \\
\text { Gêner le porteur de balle }\end{array}$ \\
\hline
\end{tabular}

Quelques actions significatives sont définies et présentées dans le tableau III. En fonction du contexte, de la situation de jeu et du niveau des joueurs, trois actions positives (AP) seront choisies pour l'observation. On peut donc calculer le rapport : actions significatives / nombre de minutes de jeu (Marle, Zerai, \& Gréhaigne, 2011).

Les premières études montrent que pour cinq minutes de jeu, un rapport de 0.7 ou 0.8 atteste d'une transformation dans les comportements des joueurs. D'un point de vue pratique, l'enseignant peut gérer l'observation des différents joueurs (jusqu'au 5x5) avec l'aide d'un secrétaire. La focalisation essentiellement sur le porteur de balle ne pose guère de problème ; l'utilisation de jeux réduits, sur terrains adaptés, devrait permettre à tous les joueurs d'entrer très souvent en contact avec le ballon. Enfin, les résultats de ce type d'observation peuvent être transmis quasiment immédiatement aux élèves.

Pour comparer les joueurs avec des temps de jeu différents, le rapport actions positives / nombre de minutes de jeu est intéressant.

\section{Données chiffrées au niveau collectif}

Dans un rapport de forces équilibré (ce qui oblige l'enseignant à « construire » ce rapport de forces) et pour des séquences de jeux réduits $(3 \times 3-4 \times 4-5 \times 5)$, nous allons faire deux propositions. Tir / minute de jeu

Le nombre de buts marqués et le nombre de tirs «cadrés » (on peut par convention prendre en compte les tirs réellement cadrés plus les tirs passant à moins d'un mètre des buts), ramenés à la minute de jeu. Ce rapport tirs + buts / minute donne une indication précise sur le niveau de jeu. Ce rapport s'élève avec le niveau de jeu. Au niveau scolaire pour les équipes les plus débrouillées, ce rapport peut aller jusqu'à 3. Pour les équipes avec des joueurs en grande difficulté, il peut être inférieur à 1 .

Tir + but / balle jouée 
Ce deuxième indice concerne le nombre de tirs + nombre de buts / nombre d'entrées en possession du ballon. Cela renseigne sur les entrées en possession du ballon qui donnent lieu à une séquence complète de jeu jusqu'à un tir. Les pertes du ballon étant fréquentes, il est difficile à observer lorsque le niveau de jeu est faible. Ce rapport peut varier de 10 à $15 \%$ pour les équipes faibles à 60 ou $65 \%$ pour les équipes fortes.

\section{Données chiffrées au niveau individuel}

La contribution de chaque joueur au déroulement des séquences de jeu constitue un autre indicateur pertinent pour évaluer la prestation de celui-ci.

Toujours dans un rapport de forces équilibré et pour des séquences de jeux réduits $(3 \times 3,4$ x 4 ou 5 x 5), nous allons également faire deux types de propositions (cf. Marle, Pasteur \& Voland, 1996).

\section{Des données chiffrées}

Il est possible de relever un certain nombre de données pour chacun des joueurs (Tableau II) et de calculer des rapports d'efficacité.

Tableau II. Exemple de fiche individuelle complète de recueil de données

\begin{tabular}{|c|l|l|l|}
\hline Nom du joueur : & Match $\mathrm{n}^{\circ}:$ & \multicolumn{2}{l|}{ Classe ou groupe : } \\
\hline Activité du joueur & & TOTAL \\
\hline Balles reçues (BR) & & \\
\hline Balles conquises (BC) & & \\
\hline Balles jouées ou touchées (BJ) & Balles reçues + balles conquises (BR + BC) & \\
\hline Balles distribuées (BD) & & \\
\hline Balles perdues (BP) & & \\
\hline Tirs tentés (T) & & \\
\hline Buts marqués (B) & & \\
\hline
\end{tabular}

Nous allons maintenant exposer quelques définitions et caractéristiques de ces données chiffrées pour bien comprendre les utilisations possibles de ces indicateurs. Cet outil constitue un inventaire très détaillé des événements qui peuvent subvenir dans le jeu. Il n'est pas à utiliser forcément dans 
sa totalité (en entier) car en fonction de ce qui est enseigné, on peut se construire une grille d'évaluation spécifique simplifiée avec tout ou partie des données exposées.

Nous allons définir précisément ces données chiffrées pour bien comprendre les utilisations possibles de ces indicateurs d'efficacité.

- Les balles jouées (BJ) ou balles touchées en football (BT) sont constituées par l'ensemble des balles touchées ou jouées par un joueur. Ici, le nombre de balles jouées renseigne sur les entrées en possession du ballon d'une équipe ou d'une joueuse dans un temps donné. Ce nombre indique aussi le volume de jeu et nous informe sur l'évolution de la circulation de balle entre les partenaires ainsi que le nombre d'échanges de balle réussis. Chez les débutants, plus le nombre de balles jouées est important, plus le jeu est de qualité dans une unité de temps donné. Dans certains cas, il peut également être un indicateur de domination.

- Concernant les balles conquises (BC), la joueuse regagne volontairement la possession de la balle soit grâce à un duel victorieux ou une interception : son équipe devient attaquante.

- Les balles perdues (BP) ce sont les balles perdues en défense, en attaque ou sur fautes... ainsi que les tirs ratés non récupérés par l'équipe attaquante en basket-ball ou au handball. C'est donc quand l'équipe perd la possession du ballon au profit de ses adversaires. Ces pertes de balle proviennent principalement d'erreurs techniques ou tactiques pour l'équipe en possession du ballon, mais aussi de rapports d'opposition mal maîtrisés dans le cas de la récupération active de la balle par l'équipe adverse. Dans un cycle de travail, « diminuer le nombre de balles perdues et augmenter le nombre de buts marqués » constitue souvent un objectif prioritaire

- Tirs (T) Ce sont les tirs au but tentés.

- Buts (B) Ce sont les buts marqués.

\section{- Données chiffrées pour l'équipe dans rencontres successives}

Nous avons choisi par souci de cohérence de présenter les résultats des mêmes affrontements entre des équipes de filles du lycée de Gray de classe de première jouant en jaune et en bleu. Les rencontres de handball sont de 2 fois 15 minutes à 5 contre 5 (Caty, Zerai, \& Gréhaigne, 2009). L'évolution du jeu et les progrès constatés après onze leçons sont marquants. Au début du cycle, beaucoup d'élèves avaient peur du ballon et se positionnaient de façon à ne pas le recevoir. Un certain nombre de joueuses se contentaient de faire des aller-retours entre les cibles, les pertes de balle étaient fréquentes et les résultats chiffrés décevants.

Nous traiterons successivement les résultats des joueuses en analysant les balles jouées, les balles conquises, les balles perdues, l'indice de conservation, l'indice de la défensive et enfin l'indice de la marque dans les différents tests. Nous présentons dans les tableaux de III à V l'ensemble des 
données chiffrées recueillies lors des leçons 1,6 et 12 . Il y a donc 5 leçons entre chaque test, le travail s'étalant sur 13 leçons.

Tableau III. Résultats des équipes jaunes et bleues lors de la première rencontre.

\begin{tabular}{|c|c|c|c|c|c|c|c|c|c|c|c|c|}
\hline & \multicolumn{6}{|c|}{ Équipe jaune } & \multicolumn{6}{|c|}{ Équipe bleue } \\
\hline & J 1 & $\mathrm{~J} 2$ & J 3 & $\mathrm{~J} 4$ & J 5 & Total & B 1 & B 2 & B 3 & B 4 & B 5 & Total \\
\hline Balles jouées & 24 & 15 & 7 & 6 & 13 & 65 & 18 & 20 & 6 & 16 & 6 & 66 \\
\hline Balles conquises & 4 & 1 & 1 & 0 & 4 & 10 & 2 & & 1 & 1 & 3 & 7 \\
\hline Balles perdues & 4 & 1 & 1 & 0 & 4 & 10 & 1 & 1 & 4 & 0 & 0 & 6 \\
\hline Tirs & 0 & 1 & 5 & 3 & 1 & 10 & 0 & 5 & 0 & 5 & 1 & 11 \\
\hline Buts & 0 & 4 & 1 & 0 & 0 & 5 & 0 & 4 & 0 & 0 & 1 & 5 \\
\hline
\end{tabular}

Tableau IV. Résultats des équipes jaunes et bleues lors de la deuxième rencontre.

\begin{tabular}{|c|c|c|c|c|c|c|c|c|c|c|c|c|}
\hline & \multicolumn{6}{|c|}{ Équipe jaune } & \multicolumn{6}{|c|}{ Équipe bleue } \\
\hline & $\mathrm{J} 1$ & $\mathrm{~J} 2$ & J 3 & $\mathrm{~J} 4$ & $\mathrm{~J} 5$ & Total & B 1 & B 2 & B 3 & B 4 & B 5 & Total \\
\hline Balles jouées & 12 & 17 & 12 & 15 & 11 & 67 & 18 & 17 & 15 & 10 & 10 & 70 \\
\hline Balles conquises & 2 & 2 & 4 & 1 & 2 & 11 & 2 & 2 & 3 & 1 & 1 & 9 \\
\hline Balles perdues & 2 & 9 & 4 & 1 & 1 & 17 & 5 & 2 & 0 & 1 & 0 & 8 \\
\hline Tirs & 3 & 0 & 2 & 0 & 0 & 5 & 7 & 3 & 4 & 1 & 2 & 17 \\
\hline Buts & 1 & 0 & 0 & 0 & 0 & 1 & 4 & 1 & 0 & 0 & 1 & 6 \\
\hline
\end{tabular}


Tableau V. Résultats des équipes jaunes et bleues lors de la troisième rencontre.

\begin{tabular}{|c|c|c|c|c|c|c|c|c|c|c|c|c|}
\hline & \multicolumn{6}{|c|}{ Équipe jaune } & \multicolumn{6}{|c|}{ Équipe bleue } \\
\hline & $\mathrm{J} 1$ & $\mathrm{~J} 2$ & J 3 & J 4 & J 5 & Total & B 1 & B 2 & B 3 & B 4 & B 5 & Total \\
\hline Balles jouées & 21 & 20 & 17 & 10 & 17 & 85 & 23 & 16 & 14 & 22 & 13 & 88 \\
\hline Balles conquises & 2 & 0 & 3 & 2 & 0 & 7 & 3 & 0 & 5 & 3 & 4 & 15 \\
\hline Balles perdues & 12 & 2 & 3 & 0 & 0 & 17 & 8 & 0 & 0 & 8 & 0 & 8 \\
\hline Tirs & 0 & 2 & 4 & 6 & 8 & 20 & 2 & 5 & 0 & 2 & 3 & 10 \\
\hline Buts & 0 & 1 & 0 & 5 & 2 & 8 & 1 & 4 & 0 & 1 & 0 & 6 \\
\hline
\end{tabular}

Les balles jouées

Nous analyserons tout d'abord les balles jouées dans chaque équipe lors de la troisième rencontre afin d'avoir des résultats après une familiarisation importante avec le jeu (Figure 2). Tout d'abord, les effectifs de balles jouées pour chaque équipe lors des trois rencontres ont augmenté, passant de 65 à 85 pour les Jaunes et de 66 à 88 pour les Bleues. En cette leçon 12, le jeu est plus fluide et la continuité du jeu est plus assurée car il y a moins de sorties hors du terrain de la balle, la conservation du ballon ayant progressé.

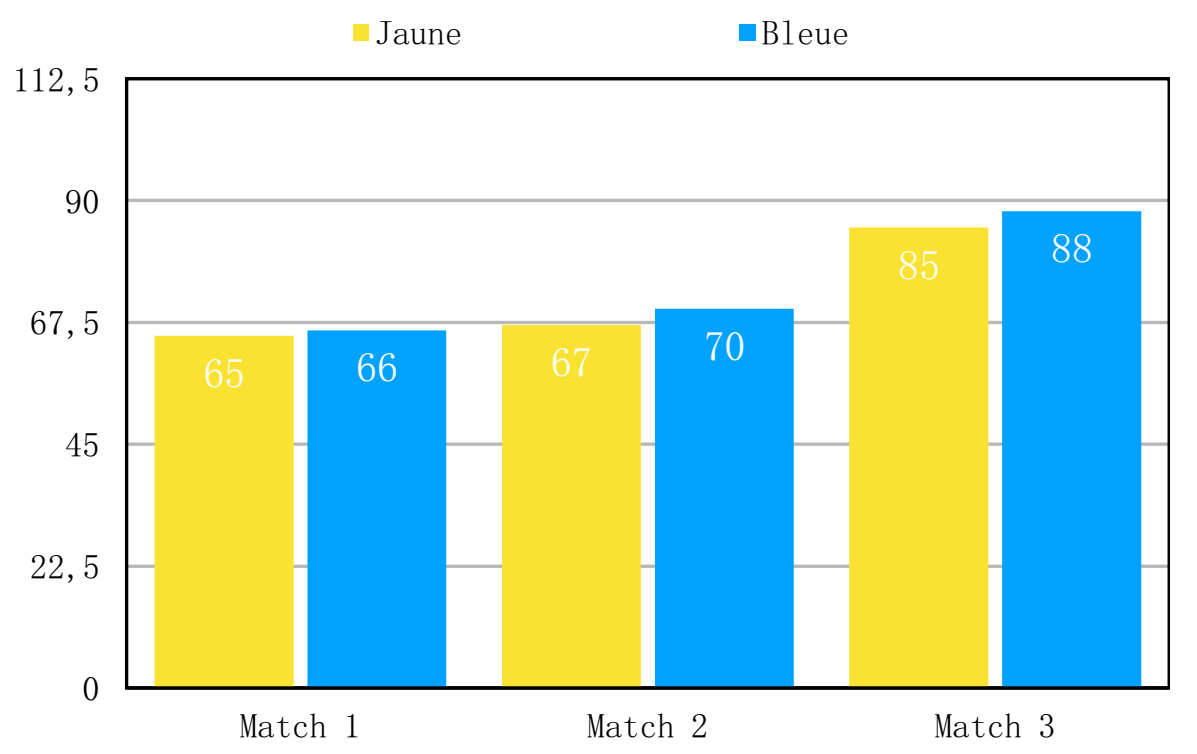

Figure 2. Répartition des balles jouées lors des trois rencontres

En ce qui concerne la répartition des balles jouées à l'intérieur des deux équipes, on peut constater une répartition mais assez équitable puisque l'on va de 10 balles jouées pour la Jaune J4 à 23 balles pour la joueuse Bleue J1. Ce fait souligne une évolution positive chez l'ensemble des joueurs. 
Chez les Jaunes, le nombre des balles jouées passe de 65 balles durant la première rencontre à 67 pour la deuxième et 85 durant la troisième. Pour les Bleues, ce nombre passe de 66 pour le premier match à 70 pour le deuxième et enfin à 88 balles pour le dernier.

Une observation plus approfondie des tableaux de résultats montre que cette augmentation n'est pas constante pour toutes les filles de la même équipe ; c'est le cas de J1 de l'équipe Jaune. Pour cette joueuse, le nombre des balles jouées diminue entre le premier et le deuxième match : il passe de 24 à 12 alors qu'une augmentation aura lieu lors de dernier match, le nombre de balles jouées atteint alors 21 .

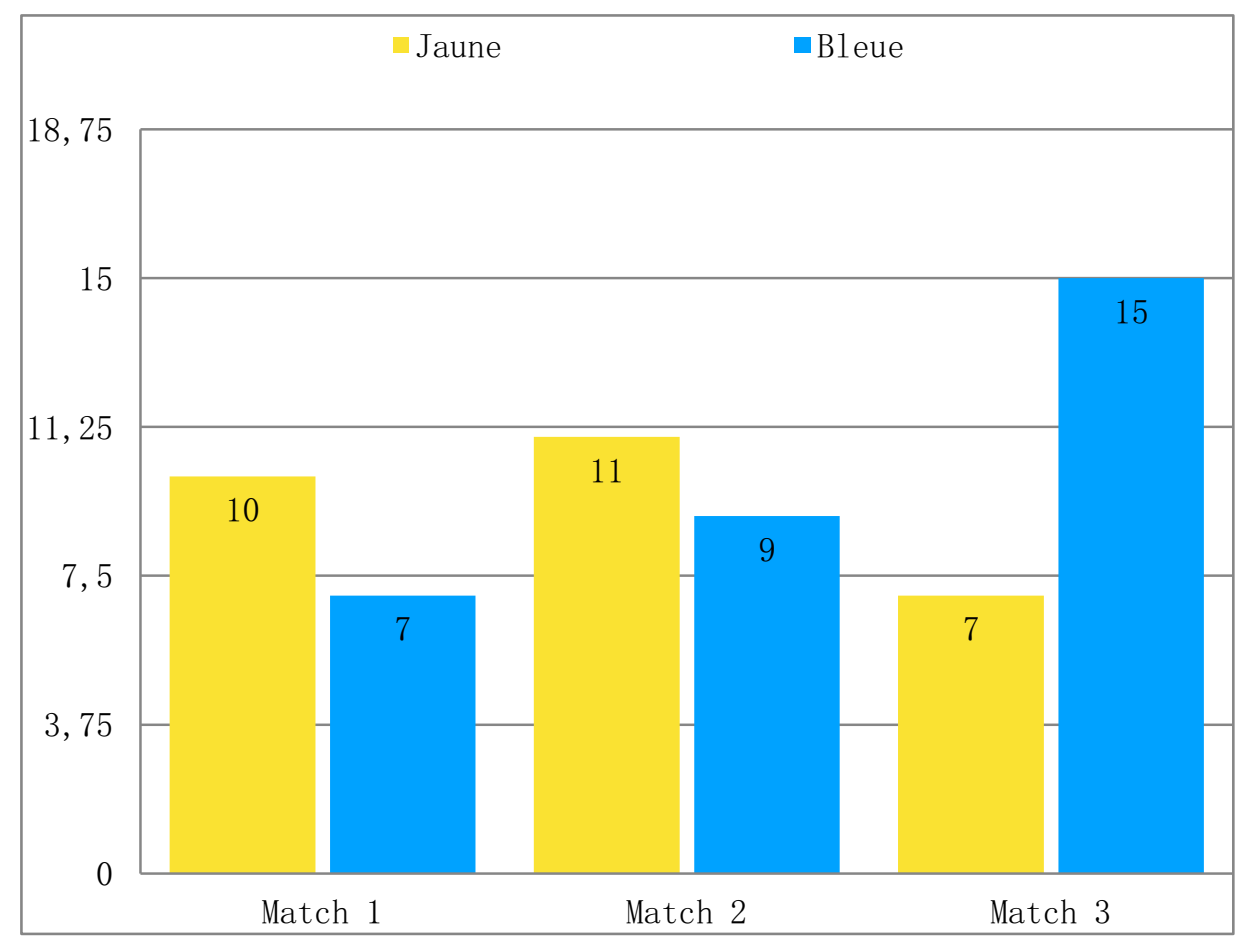

Figure 3. Répartition des balles jouées à l'intérieur de l'équipe jaune et de l'équipe bleue durant les trois rencontres

On remarque que le nombre de balles jouées des Bleues est supérieur à celui des Jaunes, mais que l'augmentation est plus importante chez les Jaunes. Cela nous permet de supposer que, malgré la domination des Bleues, l'équipe Jaune a pu établir une stratégie de jeu sur le terrain et minimiser la difficulté à gérer le rapport d'opposition. 
Les balles conquises

Il s'agit des entrées en possession du ballon où le ballon change de camp, entraînant chez les joueuses, le passage d'attaquantes à défenseurs pour les unes et de défenseurs à attaquantes pour les autres, illustrant ainsi la notion de réversibilité (Figure 4).

Le nombre de balles conquises passe de 10 à 11 puis redescend à 7 pour l'équipe Jaune alors que pour l'équipe Bleue, ce nombre est de 7 pour le premier match, de 9 pour le second et de 15 pour le dernier match. Ceci nous renseigne sur l'incapacité des filles de l'équipe Bleue à dépasser la défense de l'équipe adverse. La variation des balles conquises n'est pas constante chez la majorité des joueuses de l'équipe Jaune. Pour la Jaune J1, ce nombre est faible (4) lors de la première rencontre, diminue pendant la deuxième rencontre pour augmenter de façon spectaculaire durant la troisième séance (4, 2 et 12$)$. Par contre pour la Jaune $\mathrm{J} 2$, cette variation est inverse.

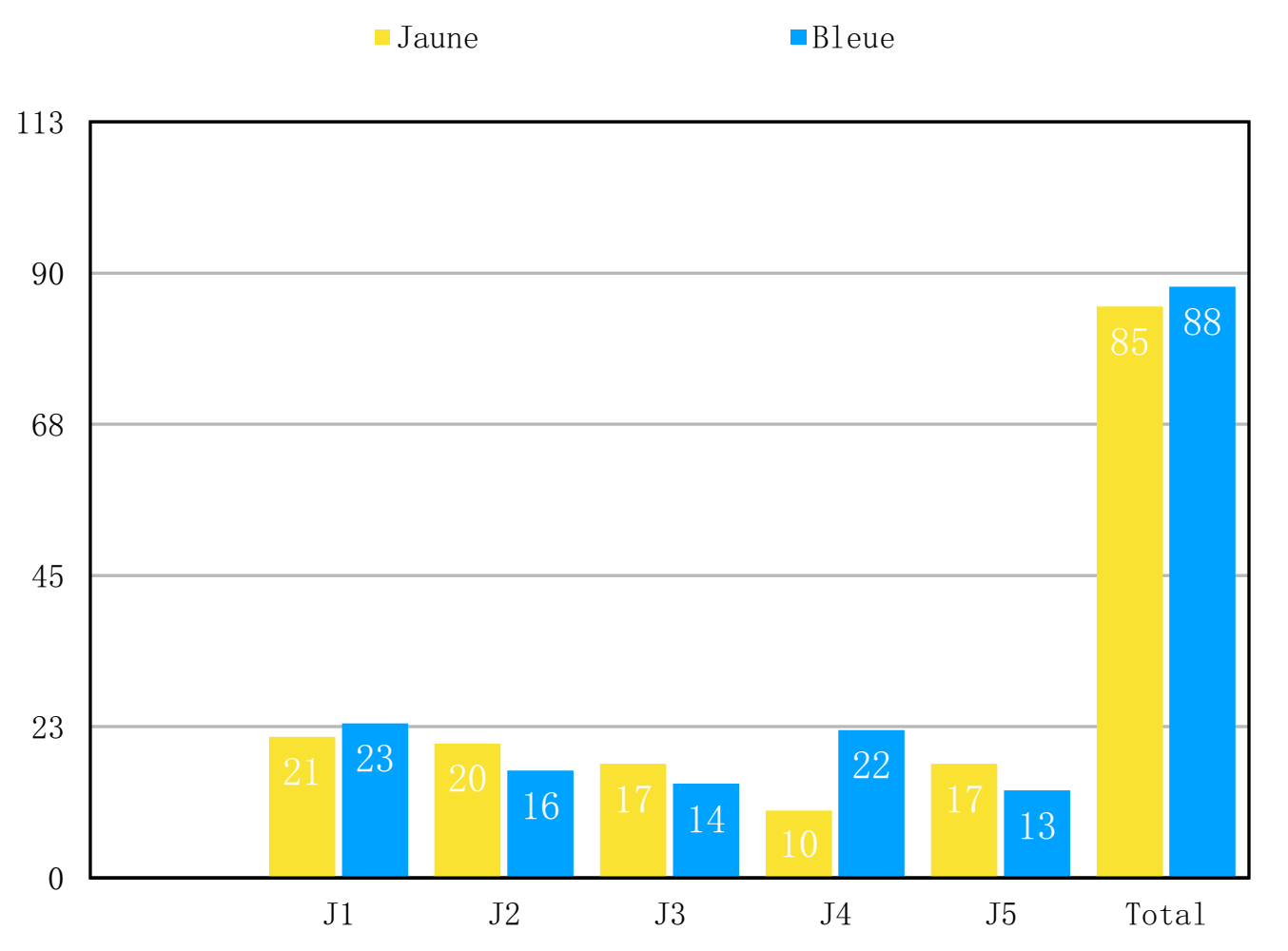

Figure 4. Répartition des balles conquises de l'équipe Jaune et de l'équipe Bleue durant les trois rencontres.

Apparaît une augmentation entre le premier et le deuxième match où le nombre passe de 1 à 9 , alors qu'entre la deuxième et la troisième rencontre, on remarque une diminution, les balles conquises passant à 2 . 
Les balles perdues.

Il s'agit des pertes de possession du ballon, le ballon change de camp entraînant le passage d'attaquantes à défenseurs des joueuses.

Pour l'équipe Jaune, le nombre de balles perdues varie puis se stabilise. Il passe de 10 à 17 balles au second match et reste à 17 pour le dernier match alors que pour l'équipe Bleue, on remarque une croissance continue : de 6 à 8 et ensuite reste à 8 balles (Figure 5). Le nombre de balles perdues des jeunes expliquent sans doute en grande partie leur défaite, la récupération haute du ballon étant un facteur important de domination. (Duprat, 2005). Fréquemment, dans ce dernier cas, la défense n'est plus en «barrage » mais à la «poursuite », car elle est positionnée derrière le porteur de balle, les attaquants et parfois loin de sa cible.

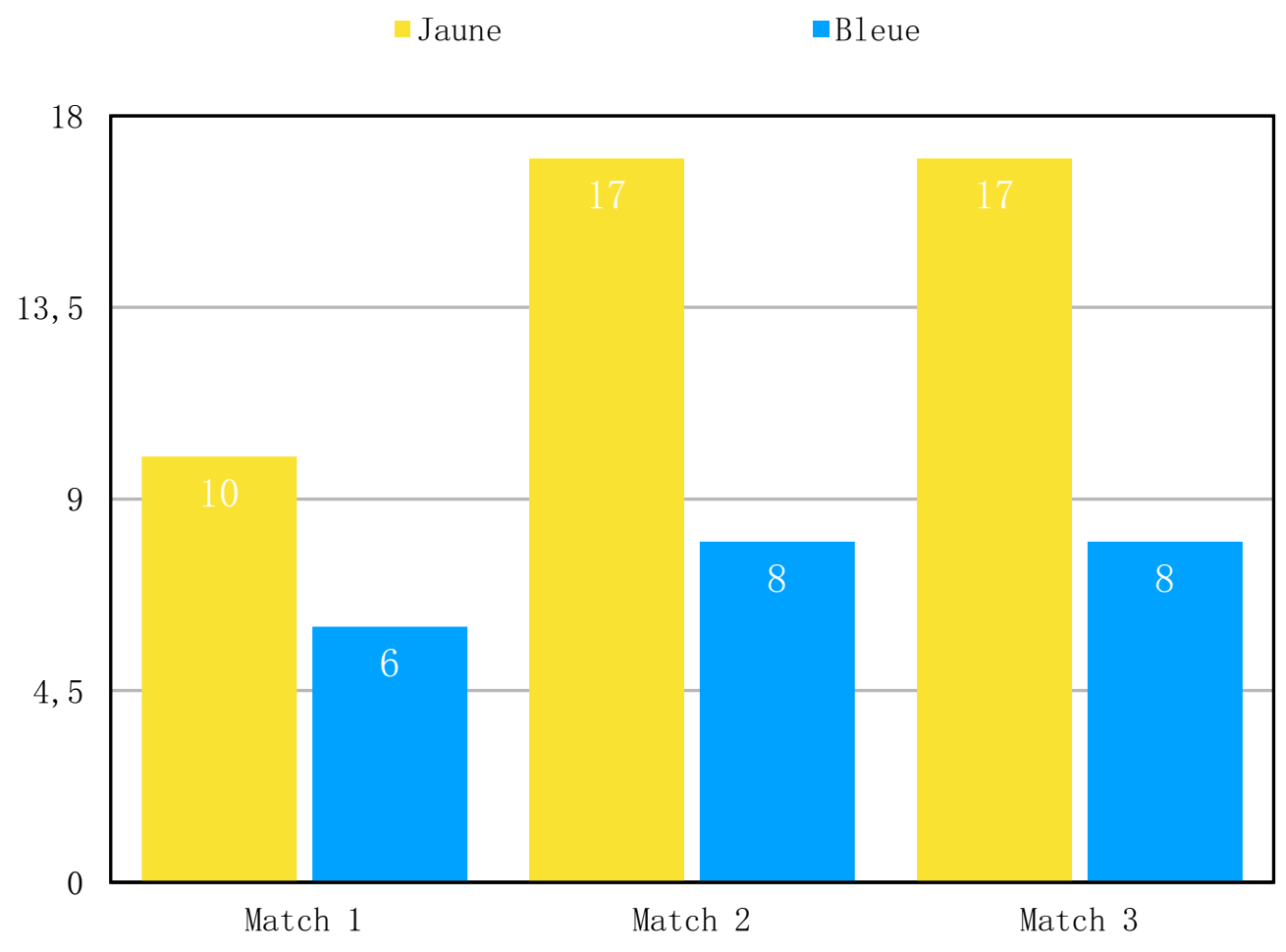

Figure 5. Répartition des balles perdues de l'équipe jaune et de l'équipe bleue durant les trois rencontres.

\section{Des indices}

Afin de mieux appréhender les évolutions dans le rapport des forces en présence, nous allons utiliser des indices mettant en rapport les données obtenues dans les trois rencontres. Nous définirons un indice comme une valeur numérique obtenue en mettant en rapport la fréquence d'apparition de deux comportements. Celui-ci ne doit pas être considéré comme une mesure absolue car le choix des éléments retenus comporte toujours une part d'arbitraire. Néanmoins, utilisés avec 
prudence, les indices constituent souvent le seul moyen d'avoir une connaissance approchée sur des phénomènes complexes et de leurs conséquences.

\section{L'indice de conservation $\left[\mathrm{I}_{\mathrm{c}}=\right.$ balles perdues/balles jouées $]$}

Les courbes de la figure 6 montrent que lors de la première et de la deuxième rencontre, la conservation de la balle est meilleure pour l'équipe Bleue. L'indice est de 0,09 , puis il passe à 0,12 alors que pour l'équipe Jaune, cet indice est de 0,1 durant le premier match et 0,16 durant le second. Lors du troisième match, les données sont inversées et l'indice devient plus faible chez les Jaunes $(0,08)$, soulignant ainsi une diminution des balles perdues dans cette équipe en relation avec l'augmentation du nombre de balles jouées. Ces courbes nous renseignent aussi sur l'amélioration du niveau de compréhension du jeu par les joueuses. Une meilleure planification de la stratégie du jeu et un progrès dans la cohésion du groupe peuvent être remarqués.

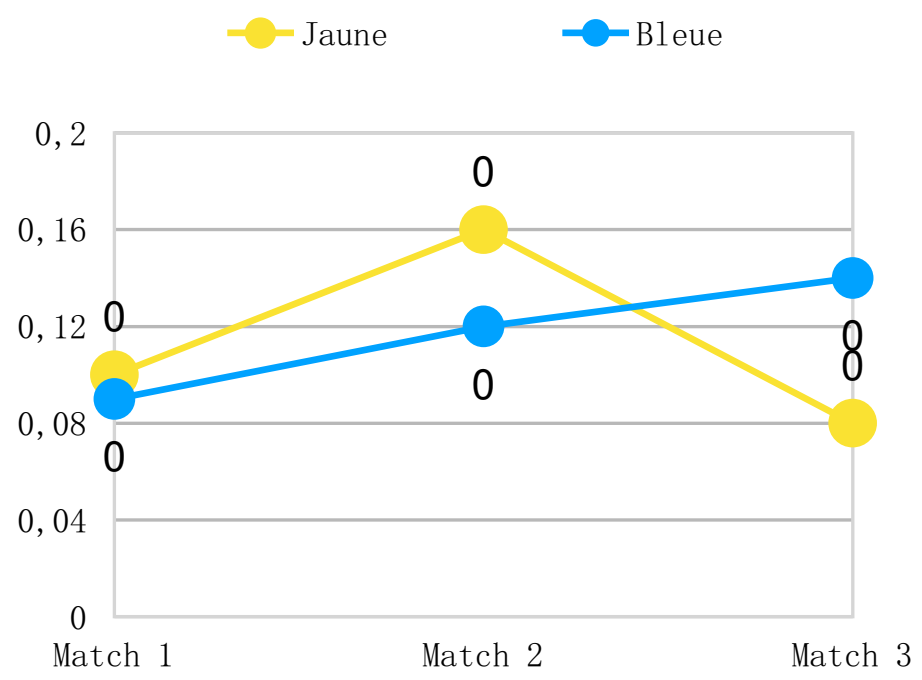

Figure 6. Indices de conservation de l'équipe Jaune et de l'équipe Bleue durant les trois rencontres.

L'indice de la défensive [ $\mathrm{I}_{\mathrm{D}}=$ balles conquises/balles perdues $]$.

Le rapport entre balles conquises et balles perdues montre l'efficacité à récupérer la balle et à la conserver grâce à une organisation adaptée en attaque et en défense. On doit se rappeler que cet indice est bon quand il est élevé (Figure 7). Pour les deux équipes, cet indice a évolué de façon opposée. 


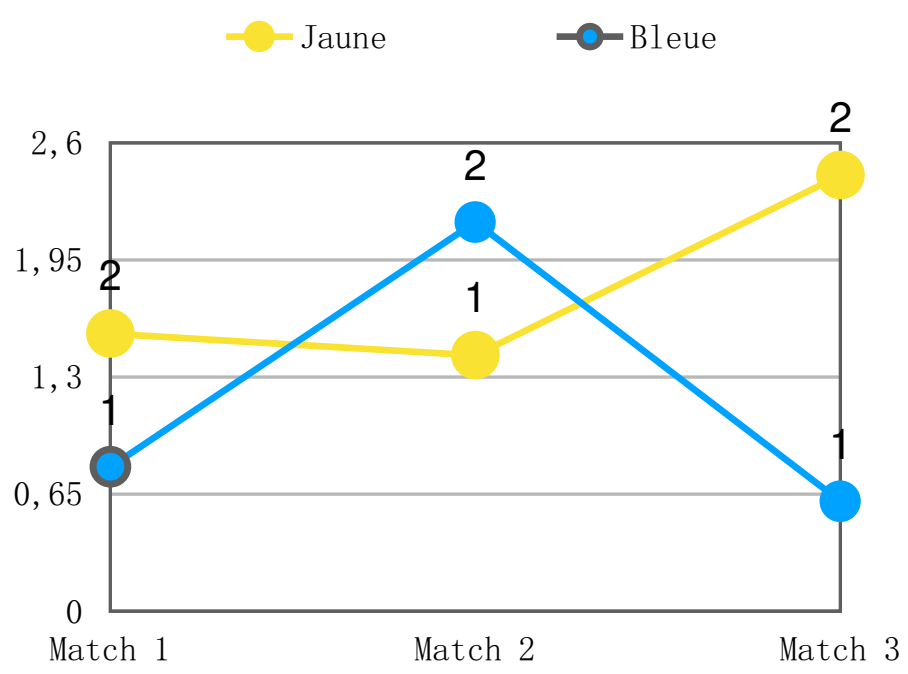

Figure 7. Indice de la défensive de l'équipe jaune et de l'équipe bleue durant les trois matchs.

Entre le premier match et le deuxième, on remarque une augmentation pour l'équipe Bleue de 0,8 à 2,16 et une diminution pour l'équipe Jaune de 1,54 à 1,42. Mais, entre le deuxième et le troisième match, ces indices évoluent inversement. On observe une diminution pour les Jaunes de 2,16 au cours du deuxième match à 0,61 pour le troisième match et une augmentation pour les Bleues où l'indice passe de 1,42 à 2,42 (Figure 6). Cet indicateur nous renseigne sur l'adaptation des joueuses au rapport de forces. La perte de la balle à la suite d'une interception est fréquemment due à une mauvaise appréciation de la situation de jeu. Par contre, cette différence pour l'équipe Jaune illustre une lecture plus efficace des informations.

\section{Capacité offensive : l'indice de la marque}

Pour l'équipe Jaune, le nombre de tirs diminue entre le premier et le deuxième match. Il passe de 10 à 5 , pour augmenter ensuite et atteindre 20 tirs lors de la dernière rencontre. Ce même phénomène est aussi valable pour le nombre de buts marqués donnant un résultat de 5 puis 1 et enfin 8 buts. Concernant l'équipe Bleue, le nombre de tirs est de 11 pour le premier match alors que le nombre de buts est de 5 . Pour la deuxième rencontre, le nombre de tirs est de 17 et le nombre de buts est de 6. Lors du troisième match, les deux nombres sont : 10 tirs et 6 buts (Figure 8). 


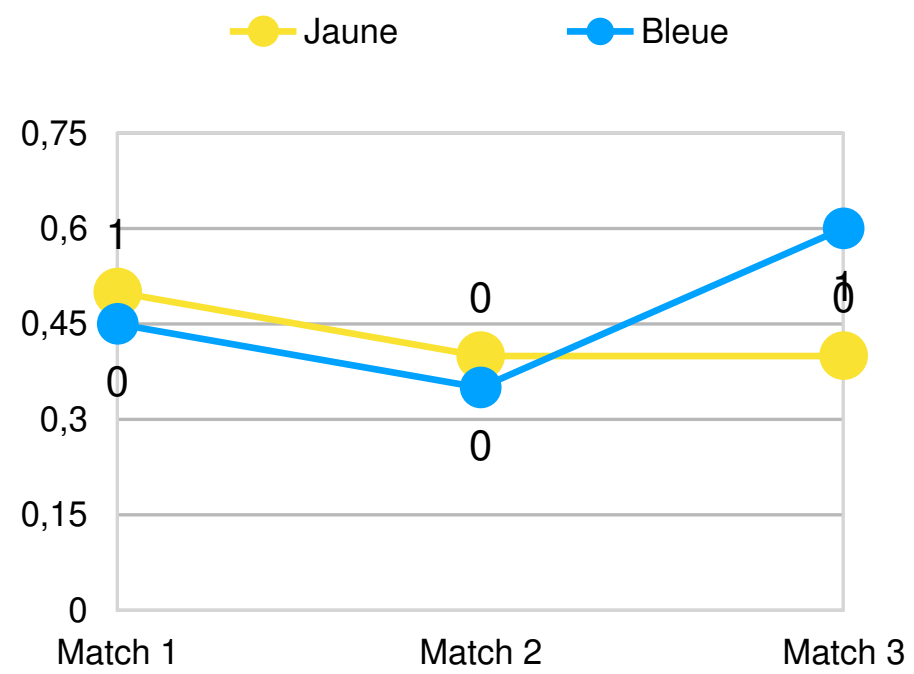

Figure 8. Indices de la marque de l'équipe Jaune et de l'équipe Bleue durant les trois rencontres.

En conclusion, un des soucis en didactique des sports collectifs est de ne pas en rester à l'analyse globale des situations d'apprentissage mais d'analyser l'activité du sujet-joueur dans son développement et sa durée. L'apprentissage a pour effet de transformer le jeu mais l'apprenant se transforme lui-même, déployant ainsi une activité construite et constructive.

Ceci permet d'avancer avec Pastré (2007) que :

- On ne peut pas dissocier activité et apprentissage car toute activité productive s'accompagne toujours d'une activité constructive, plus ou moins marquée : on apprend par l'action. Réciproquement, il faut une activité productive servant de support pour que puisse se développer une activité constructive.

- Avec des données fiables, l'activité constructive peut se prolonger bien au-delà de la fin de l'action. C'est précisément la raison pour laquelle l'analyse après coup de sa propre activité, autrement dit, l'analyse réflexive et rétrospective, est un remarquable instrument d'apprentissage.

- Il est évident que l'activité constructive permet une dé-singularisation de la situation. Elle envisage une généralisation possible, même si cette généralisation reste partielle.

\section{Des aspects qualitatifs}

Pour le football, le découpage statique d'un terrain est commode si l'on prend la précaution de le combiner avec d'autres outils. Sinon, il devient vite complètement formel, voire faux en isolant les données du cadre où elles ont été produites. L'observation topologique étudie des formes de jeu en rapport avec leur emplacement sur le terrain. Ici, l'observation la plus simple qui puisse être utilisée 
consiste à considérer d'une part la zone défensive et, d'autre part, la zone offensive. Si on divise chaque zone, on obtient quatre zones d'observation : défensive, (a), pré-défensive (b) ; préoffensive (c) et offensive (d), comme illustrées dans la figure 9.

On peut aussi considérer le corridor central du terrain et deux corridors limitrophes de chaque côté. Cela permet de relever les actions de jeu menées dans l'axe «but attaqué / but défendu » (corridor central [C3 dans la figure 9]) et d'autres dans les corridors médians (C2 et C4) ou dans les couloirs périphériques (C1 et $\mathrm{C} 5$ ). Un autre outil est constitué par l'espace de jeu direct. C'est le «fameux entonnoir » dont parlent tous les techniciens du football. Celui-ci est caractéristique de tous les jeux sportifs collectifs, interpénétrés, de signes contraires et à cibles verticales (handball, water-polo, hockey sur glace, hockey sur gazon...). Ainsi, l'espace de jeu direct permet de définir pour la défense une zone de terrain où il est dangereux de perdre la balle ou de laisser l'attaque jouer à sa guise. A partir de cette surface, les joueurs attaquants peuvent enchaîner relativement facilement sur une action de jeu direct... et se trouver en avance dans une position de marque. Dans la figure 9, l'espace de jeu direct est délimité par quatre lignes pointillées qui vont de la périphérie de la ligne centrale au coin de la ligne de but et de la surface de but. 


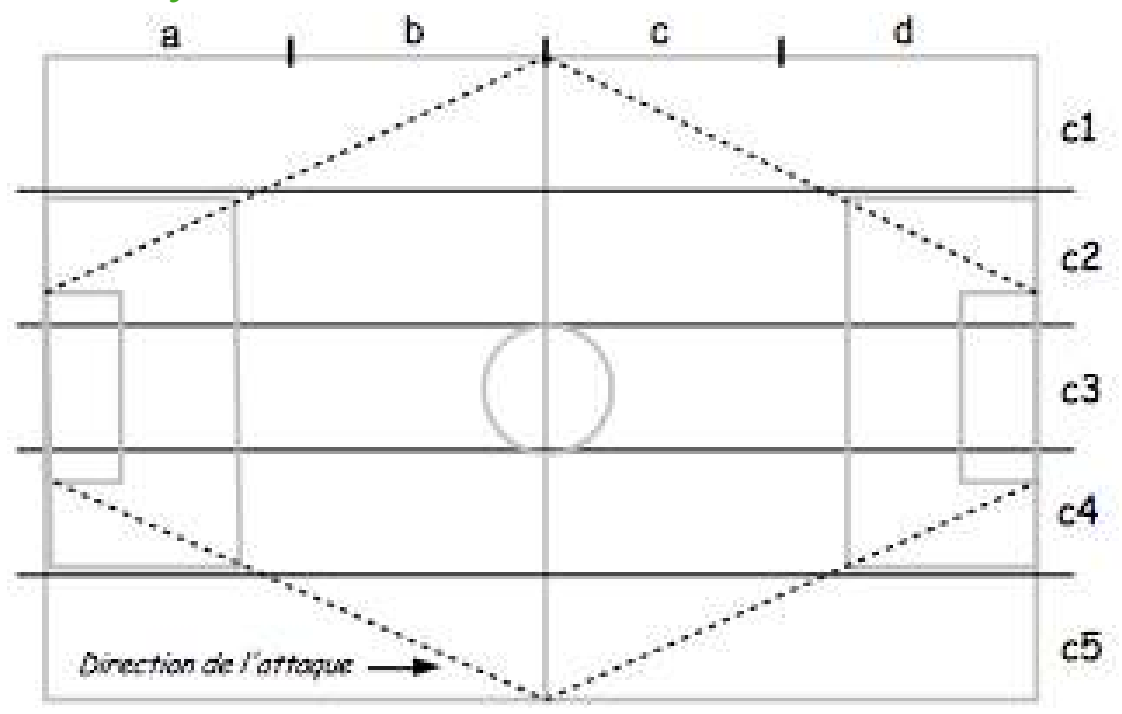

Figure 9. Différents outils statiques pour un terrain de football

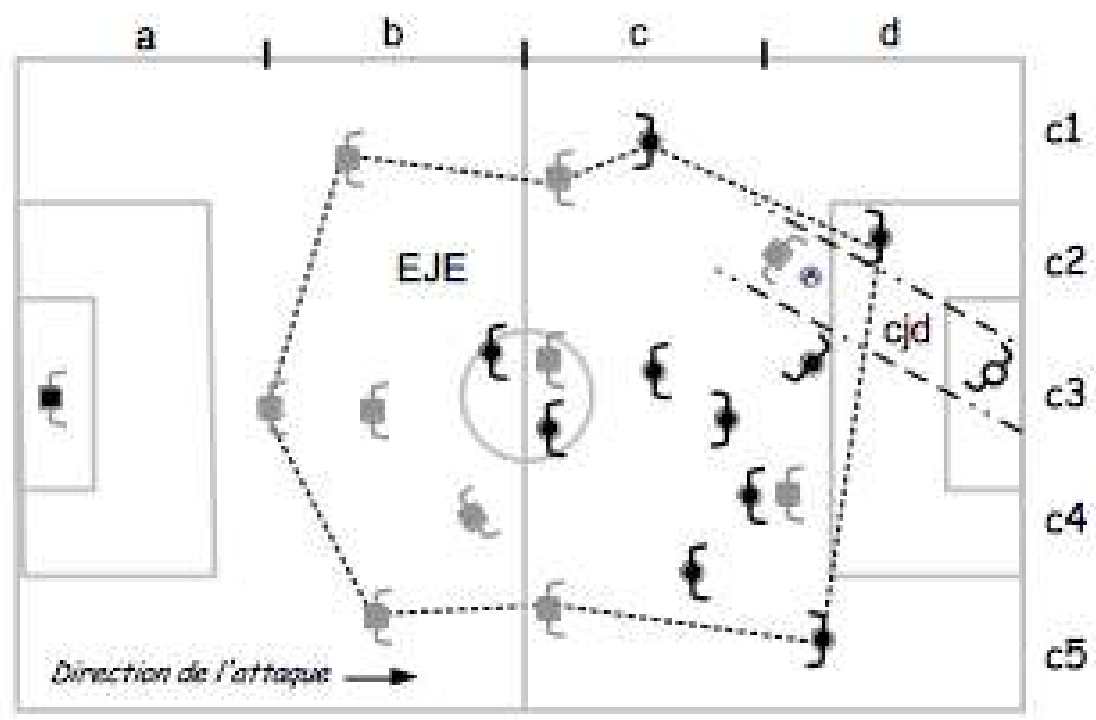

Figure 10. L'espace de jeu effectif et le couloir de jeu direct instantanés en football.

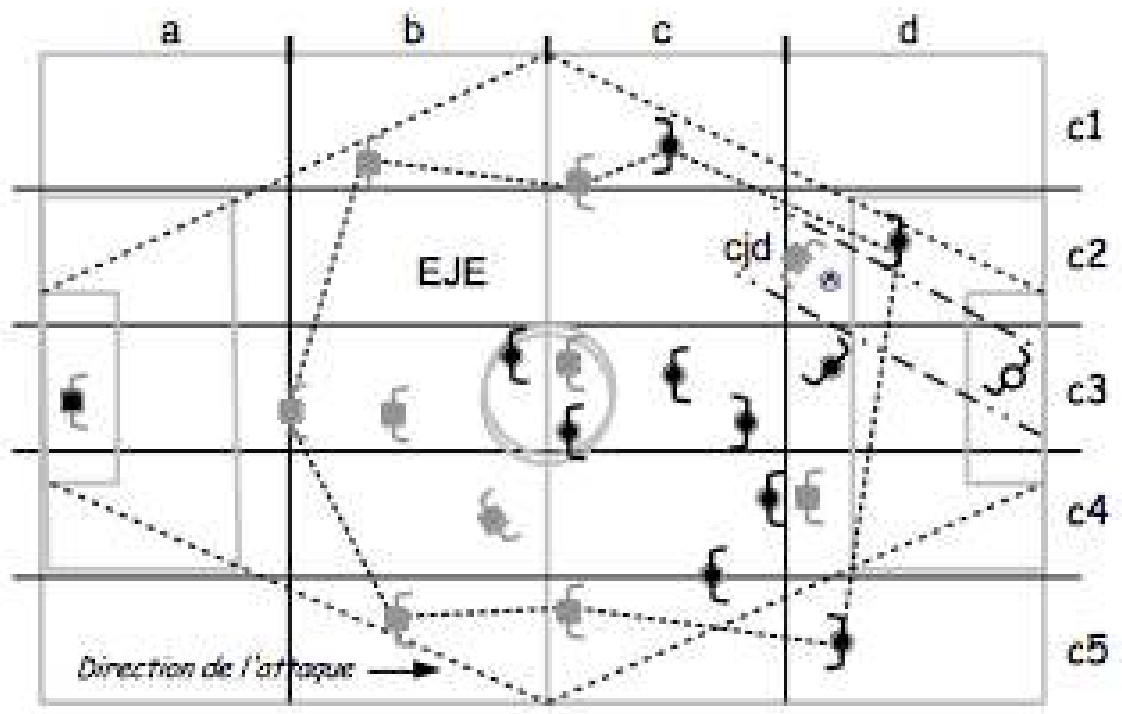

Figure 11. Les deux outils combinés pour un résultat plus précis mais plus difficile à lire. 
Pour leur part, les notions de « centre de gravité » et de «place effective sur le terrain » illustrent la façon dont un coach peut utiliser des données d'observation statiques du jeu. Sur ce thème, on peut consulter Bourbousson, Sève, \& McGarry (2010) ; Frencken, Lemmink, Delleman, \& Visscher (2011) ; Gréhaigne (1992) ; Gréhaigne, Bouthier, \& David (1997); Winkler (1988). Si l'on considère une configuration de jeu donnée, on peut la résumer en utilisant la notion d'espace de jeu effectif (Mérand, 1977 ; Gréhaigne, 1989 ; Gréhaigne, Godbout \& Bouthier, 1997 ; Gréhaigne, Billard, \& Laroche, 1999). L'espace de jeu effectif (EJE) peut être défini comme la zone polygonale que l'on obtient en traçant une ligne qui relie tous les joueurs situés à la périphérie du jeu à un instant donné (voir la ligne noire pointillée dans la figure 9). Le ballon peut se trouver dans des positions différentes par rapport à l'espace de jeu effectif : soit en position centrale au milieu de l'espace de jeu effectif, soit dans une position sur le flanc à la périphérie gauche ou droite du terrain. Dans l'exemple illustré à la figure 9, le ballon se trouve à l'avant de l'EJE, dans le couloir c2, dans la zone pré-offensive c et détermine le couloir de jeu direct (CJD) qui est l'axe ballon - cible à tout moment.

Les aspects statiques peuvent être considérés comme un premier révélateur pertinent des contradictions nées de l'opposition auxquelles les joueurs sont soumis. La mise en relation des espaces occupés réellement par les joueurs dans une équipe constituera la première étape de l'analyse du jeu (Gréhaigne, 1995).

\section{Discussion}

Les temps d'observation et d'évaluation ne sont pas destinés à occuper les élèves pendant que les autres jouent mais ont une fonction indispensable pour l'évaluation formative, la compréhension et l'analyse du jeu. En effet, le fait d'attirer l'attention de l'élève sur tel ou tel aspect de l'affrontement fournit des régularités, des invariants, des stabilités qui pourront lui servir plus tard grâce à un enrichissement de son registre de fonctionnement. Agir (et non pas réagir), c'est d'abord prévoir les conséquences de nos projets d'actions futures sur la base de nos expériences passées. En relevant des données numériques à propos du jeu, les observateurs fournissent aussi au professeur des indications qui lui permettront d'organiser le retour d'informations et d'alimenter le débat d'idées. Cela permet au professeur de faire évoluer la situation d'apprentissage en fonction de l'adéquation entre résultats obtenus et résultats attendus. Ainsi, à l'aide d'un comparateur, cela permet la notation après construction d'un barème (Figure 12) où, malgré un réel souci d'équité chez les enseignants, on pointe souvent des dérives et des effets parfois néfastes voire paradoxaux (Merle, 2007).

Enfin, il est à noter que pour Allal, Cardinet et Perrenoud (1979), la régulation est le concept central de l'évaluation, dans le sens où toute évaluation - explicitement ou implicitement - est un moyen de 
régulation à l'intérieur d'un système de formation. Dans notre cas, c'est la régulation des interactions individuelles et collectives entre les joueuses qui est en cause en relation avec les décisions du professeur. Ici, apprendre consiste à construire des connaissances et des compétences motrices nouvelles car les joueuses ne disposent, au moment de l'évaluation de départ, que d'un faible bagage technique et de peu d'expérience.

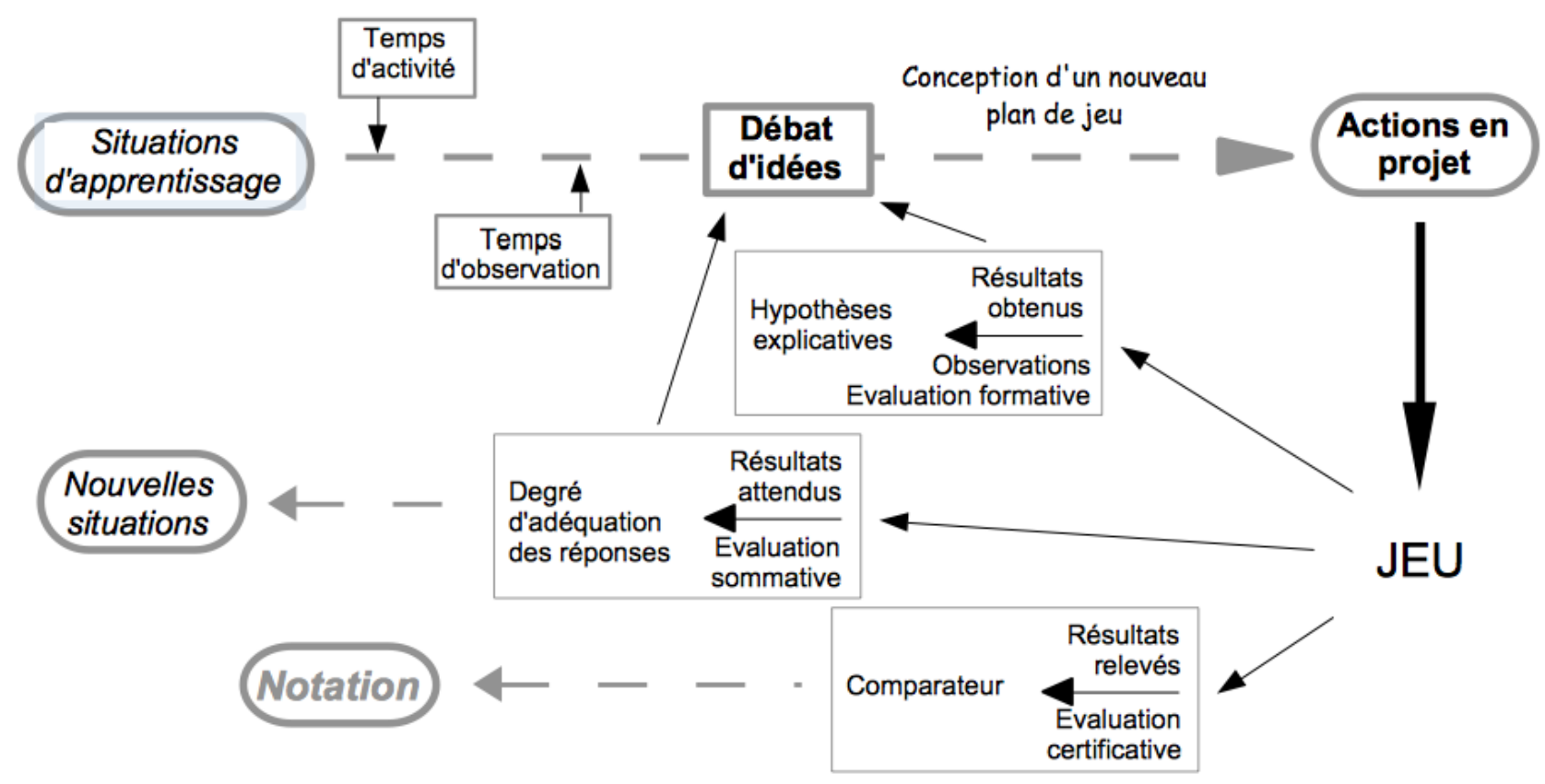

Figure 12. Différentes facettes de l'observation et de l'évaluation au cours du jeu.

Evaluer les compétences acquises consiste à proposer une ou des situations d'opposition qui nécessiteront, de la part de l'élève, une adaptation en vue d'une production pour résoudre la situation jeu (De Ketele \& Gerard, 2005). L'évolution de ces compétences motrices et de ces connaissances tactiques ne reste pas isolée, car ces ressources entretiennent d'étroites relations avec les autres connaissances (informations sur le jeu, savoir-faire sociaux, connaissances sur soi, etc.). Dans cette expérience, en liaison avec l'évaluation sommative, on a constaté une réelle progression dans les quatre équipes après onze leçons. Cela illustre un des acquis de la recherche en didactique des sports collectifs à l'école qui a consisté à montrer que l'apprentissage et l'observation d'un jeu ne peuvent se concevoir sans une pratique importante et longue.

Cela confirme les recherches menées par Berchebru (2007) ; Chang (2006) ; Gréhaigne (2011); Zerai (2006) qui ont montré qu'une durée de six à huit leçons est la période minimale pour de réels apprentissages en jeu en relation avec une observation et une évaluation du jeu qui commencent à évoluer de façon significative. Avant cette période, des réponses nouvelles ne font juste qu'émerger chez les élèves face aux problèmes vécus dans les situations de jeu. Ces réponses demandent du temps, de la pratique et de la réflexion pour devenir des réponses stabilisées qui seront disponibles 
comme ressources pour le joueur dans les cycles à venir. Certaines de ces ressources pourront ainsi être réutilisées ou reconstruites dans d'autres sports collectifs. A terme, nous devrions arriver à la conclusion qu'un objectif majeur de notre programme d'éducation physique et sportive scolaire devrait être le développement «de joueur réflexif » qui puisse utiliser leurs connaissances et leurs compétences tout au long de leur vie physique.

\section{Remarques conclusives}

L'utilisation d'indices nous semble prometteuse dans la mesure où elle permet d'installer l'évaluateur et le joueur sur la même base et ainsi dépasser une des contradictions inhérentes aux sports collectifs : apprécier un individu dans un collectif confronté à des individus dans un autre collectif aux intérêts antagonistes. Dans les sports collectifs, il y a ambivalence entre le rôle de joueur en tant que personne isolée et le statut de ce même joueur intégré à un groupe de production où la finalité est de gagner la rencontre. L'ensemble des outils exposés dans cet article vise à la production des données plus authentiques concernant l'évaluation en sport collectif. Comme nous venons de le voir, pour apprécier le travail et la participation de chacun dans une équipe, il suffit bien souvent de choisir un élément discriminant pour obtenir rapidement une photographie instantanée et intéressante des rapports de compétences entre les joueurs ainsi que du fonctionnement de l'équipe. Les données chiffrées représentent une base qui doit être développée, affinée et personnalisée en vue d'obtenir des références standardisées permettant de construire une évaluation du jeu sur des bases objectives et contextualisées.

Bien sûr, les joueurs en formation sont associés complètement à ce style d'évaluation car l'enseignant tout seul ne peut pas y arriver. Passer du temps à observer ou à relever des données est aussi, pour les élèves, une situation d'apprentissage effective où ils apprennent à décoder des comportements et à reconnaître les configurations du jeu. Enfin, cette attitude vis-à-vis de la production des acteurs nous paraît essentielle dans la perspective du développement d'une approche tactique dans l'apprentissage des sports collectifs. Il s'agit en fait de fournir aux joueurs des références objectives leur permettant de juger de la pertinence des décisions prises et de l'efficacité des actions effectuées. Ici, l'évaluateur quitte le pôle de la forme gestuelle pour se centrer sur la productivité interactive des actions des joueurs et de l'équipe. Aussi, l'éducateur prendra appui sur ces données puisées au cœur du jeu pour, d'une part, fonder ses choix didactiques et d'autre part, orienter et guider les apprentissages. En d'autres termes, les données obtenues ont bien une fonction formative car est formative toute évaluation ou observation qui a pour conséquence d'accroître l'adaptation du joueur à des situations de jeu dans la mesure où elles aident à évaluer la complexité et les transformations du jeu. Enfin, on peut constater que même si l'intention de l'évaluation est 
formative, cela ne signifie pas que le résultat sera formateur ou encore qu'il le sera pour tous les joueurs.

\section{Bibliographie}

Allal, L., Cardinet, J., \& Perrenoud, P. (Eds.) (1979). L'évaluation formative dans un enseignement différencié. Berne : Peter Lang.

Berchebru, M. (2007). Didactique de l'enseignement des sports collectifs. Quand l'utilisation de technologies multimédia et de la verbalisation améliore l'enseignement des sports collectifs. Mémoire de Mastère (non publié). Université de Franche-Comté

Blomqvist, M., Vänttinen, T., \& Luthanen, P. (2005). Assessment of secondary school students' decision making and game play ability in soccer. Physical Education and Sport Pedagogy, 10 (2), 107-119.

Bourbousson, J., Sève, C., \& McGarry, T. (2010) 'Space-time coordination patterns in basketball: Part 2. The interaction between the two teams', Journal of Sports Sciences, 28: 349-358.

Brau-Antony, S., \& Cleuziou J. P. (2005). L'évaluation en EPS. Paris : Actio

Cardinet, J. (1984). Pour apprécier le travail des élèves. Neuchâtel : IRDP.

Caty, D., Zerai, Z., \& Gréhaigne. J-F. (2009). Un cycle de handball au lycée de Gray et de Thala. In J.F. Gréhaigne (Ed.) Autour du temps Espaces, apprentissages, projets dans les sports collectifs (pp. 189-219). Besançon : Presses de l’Université de Franche-Comté.

Chang, C. W. (2006). Vers une approche constructiviste de l'enseignement du basket-ball à Tä̈wan. Thèse (non publiée). National Taïwan Normal University.

De Ketele, J.-M., \& Gerard, F.-M. (2005). La validation des épreuves d'évaluation selon l'approche par les compétences. Mesure et Evaluation en Education, 28, (3) 1-26.

Dugrand, M. (1985). Approches théoriques, expérimentale et clinique de l'enseignement du football. L'exemple au Sénégal. Thèse de troisième cycle, Université de Caen.

Dugrand, M. (1989). Le football : de la transparence à la complexité. Paris : PUF.

Duprat, E. (2005). Approche technologique de la récupération du ballon lors de la phase défensive en football, contributions à l'élaboration de contenus de formation innovants. Thèse de Doctorat (non publiée). ENS, Cachan.

Frencken, W., Lemmink, K., Delleman, N., \& Visscher, C. (2011). Oscillations of centroid position and surface area of soccer teams in small-sided games. European Journal of Sport Science, $11,215-223$.

Gréhaigne, J.-F. (1989). Football de mouvement. Vers une approche systémique du jeu. Thèse (non publiée). Université de Bourgogne. 
Gréhaigne, J.-F. (1992). L'organisation du jeu en football. Paris : ACTIO. (Collection Actualité, Recherche, Sport dirigée par Michel Laurent et Pierre Therme).

Gréhaigne, J.F. (1995). Quelques données de recherche sur les aspects tactiques dans les sports collectifs. In C.A. Hauert, L. Bizzini, \& J. Brechbûhl (Eds.), Pages choisies de sciences du sport : Psychologie - Tennis (pp. 61-83) Université de Genève : Médecine et Hygiène.

Gréhaigne, J.-F. (Ed.). (2007). Configurations du jeu, débat d'idées et apprentissage des sports collectifs. Besançon : Presses de l’Université de Franche-Comté.

Gréhaigne, J.-F. (Ed.) (2011). Des signes au sens : Le jeu, les indices, les postures et les apprentissages dans les sports collectifs à l'école. Besançon : Presses de l'Université de Franche-Comté.

Gréhaigne, J-F., \& Roche, J. (1993). Les sports collectifs au bac. Revue EP\&S, 240, 80-83.

Gréhaigne, J.-F., Billard, M., \& Laroche, J.Y. (1999). L'enseignement des jeux sportifs collectifs à l'école. Conception, construction, évaluation. Bruxelles : De Boeck.

Gréhaigne, J.-F. , Bouthier, D., \& David, B. (1997). Dynamic systems analysis of the opponent relationships in the collective actions in soccer. Journal of Sports Sciences, 15, 137-149.

Gréhaigne, J.-F., Godbout, P., \& Bouthier, D. (1997). Performance assessment in team sport. Journal of Teaching in Physical Education ,16, 500-516.

Luthanen, P. (1986) Qualitative and quantitative analysis of team games by computer. University of Jyväskylä. Finland.

Marle, P., Zerai, Z., \& Gréhaigne, J.-F. (2011). Des données, des indices indices pour observer, évaluer. Et donner du sens... In J.-F. Gréhaigne (Éd.), Des signes au sens. Le jeu, les indices, les postures et les apprentissages dans les sports collectifs à l'école (pp. 159-174). Besançon : Presses de l'Université de Franche-Comté.

Mérand, R. (1977). Considérations sur une problématique de rénovation des contenus de l'Education Physique en rapport avec les activités sportives contemporaines. In CPS de la FSGT (Ed.) L'éducateur face à la haute performance olympique (pp.05-27). Paris : Sport et Plein air.

Mérand, R. (1984). Contribution à l'évaluation des connaissances et des capacités d'analyse des activités pratiquées. In SNEP (Ed.), L'évaluation en E.P.S (pp. 206-207). Paris : SNEP.

Mérand, R. (1990). Basket-ball : Lancer ou circuler ? Rencontres pédagogiques $n^{\circ} 28$. Paris : INRP.

Merle P. (2007). Les notes, secret de fabrication. Paris : PUF.

Pastré, P. (2007). Analyse du travail et formation. Recherches en éducation, 4, 22-28.

Zerai, Z. (2006). Comment les filles apprennent-elles en handball ? Apport de la verbalisation. Mastère en didactique des Activités Physiques et Sportives. Tunis, ISEFC. 
Zerai, Z. (2011). Apprentissage du handball chez les jeunes filles tunisiennes et françaises; apport de la verbalisation. Thèse (non publiée) en Sciences du Sport. Université de FrancheComté. 